Research Article

\title{
Novel Genetic Findings in a Chinese Family with Axenfeld-Rieger Syndrome
}

\author{
Kuanshu Li, ${ }^{1,2}$ Liu Yang, ${ }^{1,2}$ Ying Liu, ${ }^{2}$ and Ding Lin ${ }^{1,2}$ \\ ${ }^{1}$ Aier School of Ophthalmology, Central South University, Changsha, China \\ ${ }^{2}$ Aier Eye Hospital, Changsha, Hunan, China \\ Correspondence should be addressed to Ding Lin; linding@csu.edu.cn
}

Received 10 January 2017; Accepted 14 May 2017; Published 13 June 2017

Academic Editor: Hermann Mucke

Copyright (c) 2017 Kuanshu Li et al. This is an open access article distributed under the Creative Commons Attribution License, which permits unrestricted use, distribution, and reproduction in any medium, provided the original work is properly cited.

\begin{abstract}
Purpose. To describe a Chinese family with Axenfeld-Rieger syndrome (ARS) and report our novel genetic findings. Methods. Nine members of the same family underwent complete ophthalmologic examinations and genetic analysis. Genomic DNA was isolated from veinal blood and amplifed using PCR; the products of PCR were sequenced and compared with FOXC1 and PITX2 genes, from which the mutations were found. Results. Through the ophthalmologic examinations, 8 subjects were diagnosed as ARS and 1 subject was normal. A homozygous mutation c.1139_1141dupGCG(p.Gly380_Ala381insGly) and a heterozygous mutation c.1359_1361dupCGG(p.Gly456_Gln457insGly) in FOXC1 were identified in all subjects. The mutation (c.-10-30T>C) was identified in PITX2 in subjects III-1 and III-3. Conclusions. We found novel gene mutations in a Chinese family with ARS, which provides us with a better understanding of the gene mutation spectrum of ARS and the assistance for the genetic counseling and gene-specific therapy in the future.
\end{abstract}

\section{Introduction}

Axenfeld-Rieger syndrome (ARS) is a rare autosomal dominant disorder, characterized by anterior segment abnormalities and systemic abnormalities $[1,2]$. Common anterior segment abnormalities include [3-5] iris hypoplasia, corectopia, polycoria, iridocorneal adhesions, posterior embryotoxon, and glaucoma. The systemic abnormalities include $[1,6]$ the cardiovascular outflow tract, midface hypoplasia, flat nasal root, maxillary and mandibular hypoplasia, hypertelorism and telecanthus, skeletal anomalies, hearing loss, dental abnormalities, and redundant periumblical skin. ARS has complete penetrance but variable expressivity [7].

To date, two major genes, forkhead box $\mathrm{C} 1$ (FOXC1) on chromosome 6p25 and pituitary homeobox 2 (PITX2) on chromosome 4q25, have been demonstrated to cause ARS. Mutations in FOXC1 and PITX2 can explain about $40 \%$ of ARS $[1,8,9]$. Mutations in CYP1B1 was identified in a child with ARS and congenital glaucoma [10]. Micheal et al. reported an ARS family caused by mutations in PRDM5 [11]. Riise et al. reported the association between ARS and
PAX6 deletion [12], but then it was found to be incorrect [13]. In addition, two other loci on chromosomes 13q14 and 16q24 have been suggested to be associated with ARS by linkage analyses, but the specific disease-causing genes have not yet been identified $[4,14]$.

In this study, we performed complete ophthalmologic examinations and analysis of FOXC1 and PITX2 of all subjects and report our novel genetic findings.

\section{Materials and Methods}

2.1. Subjects. Two generations of a Chinese family with ARS were recruited to Aier Eye Hospital of Changsha (Figure 1). The study was approved by the ethics committee of Aier Eye Hospital of Changsha and adhered to the tenets of the Declaration of Helsinki. We received informed consent from all subjects before the study.

2.2. Clinical Evaluations. We performed full ophthalmologic examinations on all subjects, including visual acuity, intraocular pressure measurements (Goldman), slit lamp, anterior 


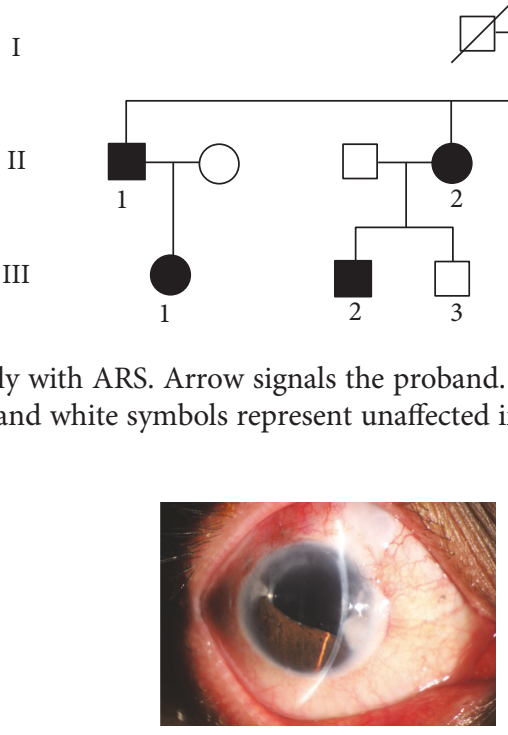

(a)

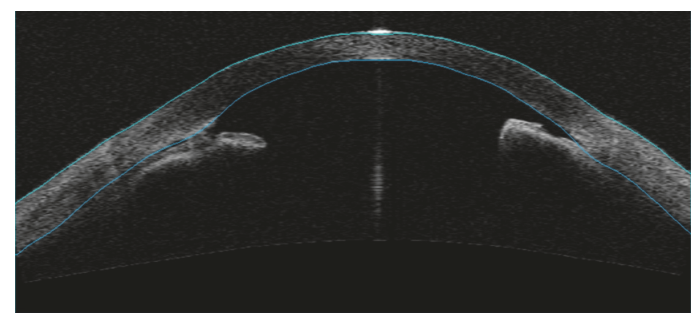

(c)

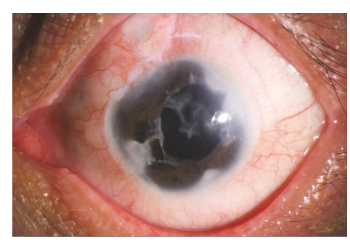

(b)

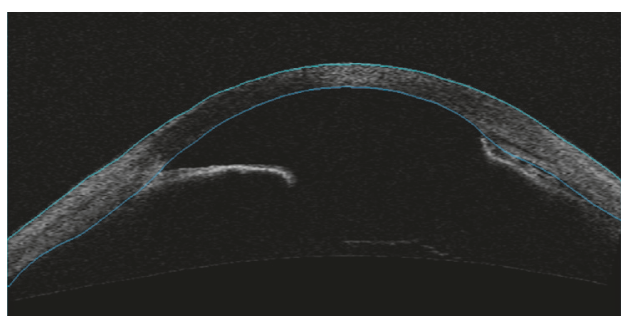

(d)

FIgURE 2: Ocular characteristics of subject II-3. Anterior segment photography showed iris hypoplasia, iridocoloboma, corectopia, and peripheral anterior synechia in both eyes $(a, b)$. Anterior segment OCT showed iridocorneal adhesions in both eyes $(c, d)$.

segment photography, visual field test (Humphrey 750, Carl Zeiss, Germany), and anterior segment OCT (Carl Zeiss, Germany). If the refractive medium was clear, we also performed funduscopy and gonioscopic and retinal nerve fiber layer (RNFL) thickness measurements (Carl Zeiss, Germany).

2.3. Mutation Analysis. About $2 \mathrm{ml}$ of venous blood was sampled from each subject and collected in vacutainer tubes (Sanjiu Medical Technology Co., Ltd., Liuyang, China) containing EDTA. Genomic DNA was extracted from each blood using a genomic DNA mini kit for blood (Life Technologies). All coding exons, with flanking intronic regions, of FOXC1 and PITX2 were amplified using PCR with primers. The amplifed DNA was purifed by agarose gel electrophoresis and sequenced on a 3730/3700xl automated DNA sequencer (Applied Biosystems).

\section{Results}

3.1. Clinical Evaluations. Through the ophthalmologic examinations, subject III-3 was normal and the other 8 subjects were diagnosed as ARS.

3.2. Subject II-3. The proband of this family is male who is 48 years old. He was referred to our hospital because of decreased visual acuity in his right eye. He underwent trabeculectomy and cataract surgery in both of his eyes at another hospital about 10 years ago; however, he had been completely blinded in his left eye. His IOP measured with Goldmann tonometry in the right eye was up to $40 \mathrm{mmHg}$. Slit lamp examination revealed iris hypoplasia, iridocoloboma, corectopia, and peripheral anterior synechia (Figure 2). No systemic abnormalities was found. We performed trabeculectomy with mitomycin $\mathrm{C}$ in his right eye. Postoperatively, the IOP was well controlled.

3.3. Subject III-1. The proband's niece is female who is 28 years old. She had no history of surgery in her both eyes. At present, she uses timolol, azopt, and alphagan to control IOP. Her IOP measured with Goldmann tonometry was $35 \mathrm{mmHg}$ in the right eye and $36 \mathrm{mmHg}$ in the left eye. Corectopia and peripheral anterior synechia were seen in the right eye, and iris hypoplasia, corectopia, polycoria, and peripheral anterior synechia were seen in the left eye (Figure 3). No systemic abnormalities was found. We advised her to have antiglaucoma surgery in both her eyes as soon as possible.

3.4. Subject III-3. The proband's nephew is male who is 31 years old. His uncorrected visual acuity was 20/20 in both eyes. IOP measured by Goldmann tonometry was $10 \mathrm{mmHg}$ in the right eye and $12 \mathrm{mmHg}$ in the left eye. Slit lamp examination revealed no anterior segment abnormalities (Figure 4). 


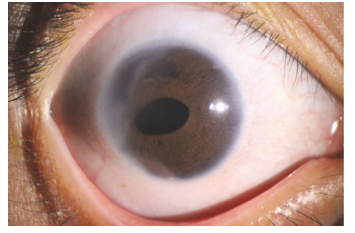

(a)

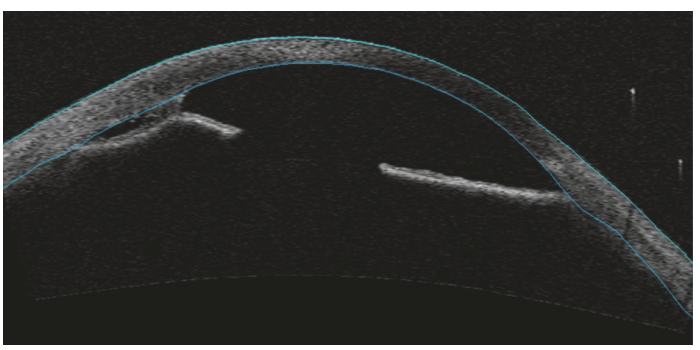

(c)

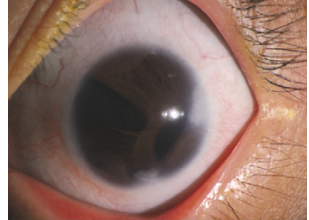

(b)

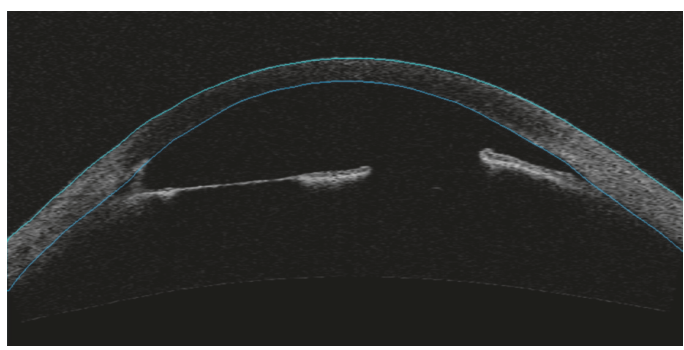

(d)

Figure 3: Ocular characteristics of subject III-1. Anterior segment photography showed corectopia and peripheral anterior synechia in the right eye (a) and iris hypoplasia, corectopia, polycoria, and peripheral anterior synechia in the left eye (b). Anterior segment OCT showed iridocorneal adhesions in the right eye (c) and iris hypoplasia and iridocorneal adhesions in the left eye (d).

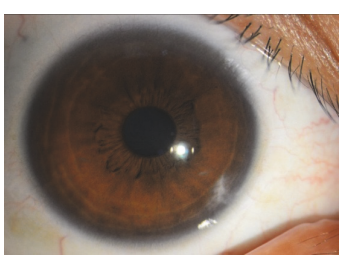

(a)

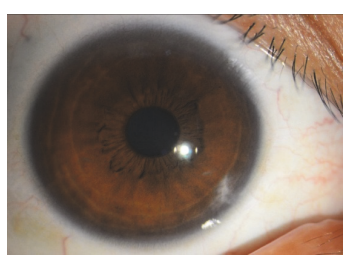

(b)
FIGURE 4: Ocular characteristics of subject III-3. Anterior segment photography showed no anterior segment abnormality in both eyes $(a, b)$.

The visual field test was normal. No systemic abnormalities was found.

3.5. Genetic Analysis of PITX2 and FOXC1. DNA sequence analysis of FOXC1 shows a homozygous mutation c.1139_1141dupGCG(p.Gly380_Ala381insGly) (Figure 5) and a heterozygous mutation c.1359_1361dupCGG(p.Gly456_Gln457insGly) (Figure 6) in all subjects.

DNA sequence analysis of PITX2 shows a heterozygous mutation c.-10-30T $>C$ in subjects III-1 and III-3 (Figure 7).

\section{Discussion}

Axenfeld-Rieger syndrome is a rare autosomal dominant disorder where phenotypes of the same mutation are variable; this is likely to be caused by environmental factors and/or modifier genes [15]. In our study, a homozygous mutation c.1139_1141dupGCG(p.Gly380_Ala381insGly) and a heterozygous mutation c.1359_1361dupCGG(p.Gly456_Gln457insGly) in FOXC1 were found in all subjects, but their clinical findings were variable. The proband showed iris hypoplasia, corectopia, and peripheral anterior synechia; subject III-1 showed corectopia and peripheral anterior synechia in the right eye and iris hypoplasia, corectopia, polycoria, and peripheral anterior synechia in the left eye, even subject III-3 showed no abnormities. This is consistent with previous reports $[16,17]$.

The two major genes of ARS are FOXC1 and PITX2. FOXC1 is a member of the forkhead family of transcription factors, which recognizes and binds to specific DNA sequences through the conserved 110-amino-acid forkhead domain (FH) and thus activates the target genes [18-20].

FOXC1 is strongly expressed in the skeletal muscle, kidney, liver, and heart and plays important roles in embryogenesis, tissue-specific gene expression, and tumor development $[20,21]$. Mutations in FOXC1 include intragenic mutations, microscopic and submicroscopic deletions, and duplications [22]. PITX2 is a member of the bicoid-like homeobox transcription factor family, which plays important roles in the genetic control of development, particularly in pattern formation and the determination of cell fate $[8,23]$. PITX2 consists of six exons and encodes a bicoid-like homeodomain transcription factor involved in embryogenesis [9]. Its expression in neural crest cells is necessary for the development of optic stalk and anterior segment [24]. PITX2 mutations include intragenic mutations, microscopic and submicroscopic deletions, and chromosome rearrangements such as translocations [22].

In our study, a homozygous mutation c.1139_1141dupGC G(p.Gly380_Ala381insGly) and a heterozygous mutation c.1359_1361dupCGG(p.Gly456_Gln457insGly) were identified in FOXC1 in all subjects. But the homozygous mutation is in the ClinVar database as rs398123611 where it is listed as a benign variant. Similarly, the heterozygous mutation is also in the ClinVar as re398123612 where it is listed as a benign/likely benign variant. The point our research deserves special attention to is that the mutation $(c .-10-30 \mathrm{~T}>\mathrm{C}$ ), 
c.11139_1141duupGCG(p. Gly380_Ala381insGly) (homozygous)

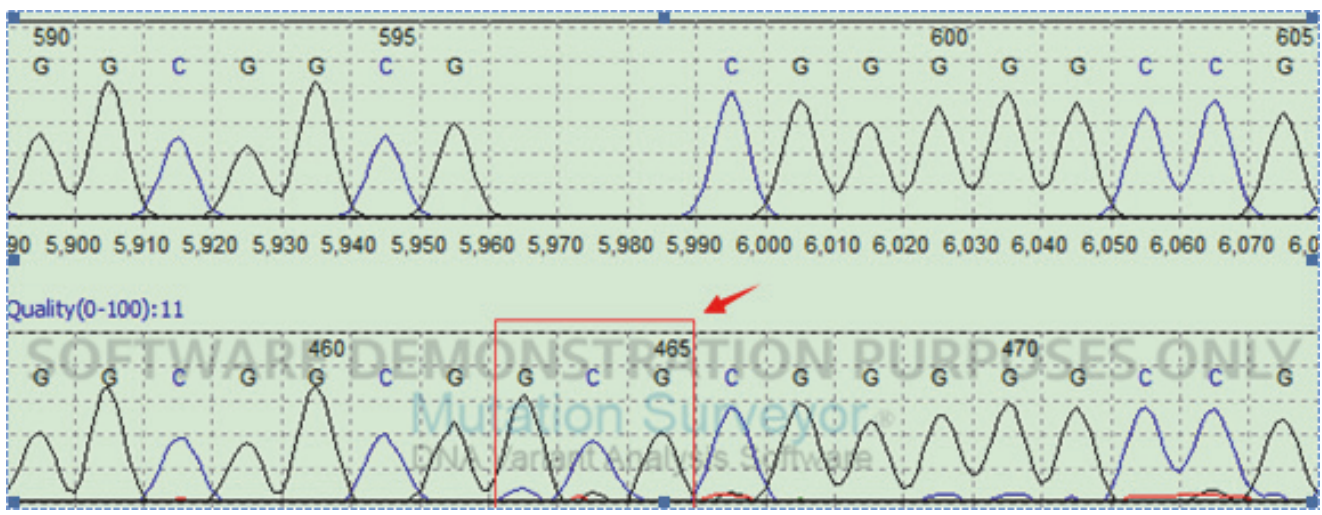

FIGURE 5: DNA sequence analysis of FOXC1 shows a homozygous mutation c.1139_1141dupGCG(p.Gly380_Ala381insGly) in all subjects.

c.1359_1361dupCGG (p. Gly456_Gln457insGly) (heterozygous)

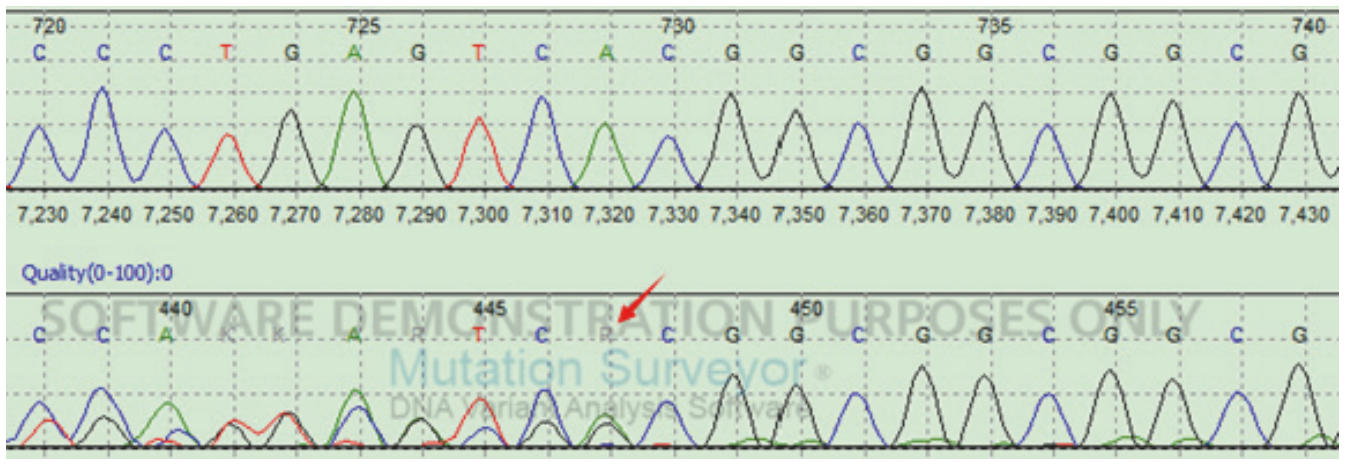

FIGURE 6: DNA sequence analysis of FOXC1 shows a heterozygous mutation c.1359_1361dupCGG(p.Gly456_Gln457insGly) in all subjects.

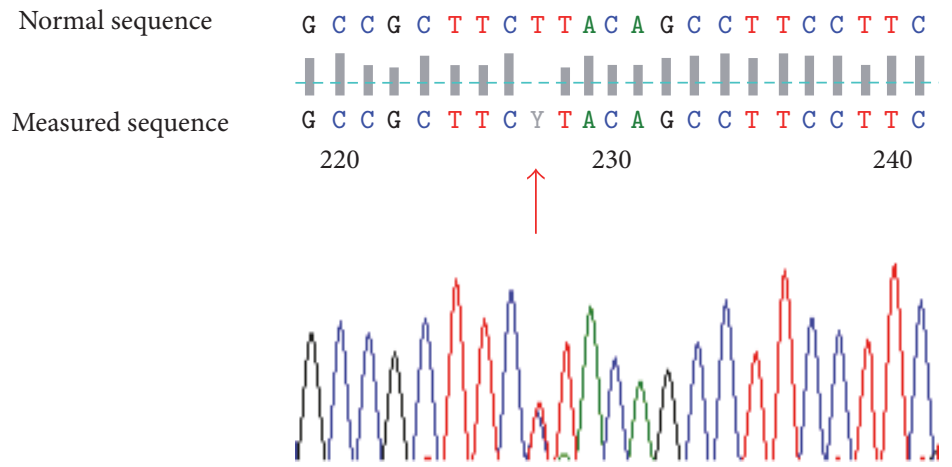

FIGURE 7: DNA sequence analysis of PITX2 shows a heterozygous mutation $c .-10-30 T>C$ in subjects III-1 and III-3.

which was identified in PITX2 in subjects III-1 and III-3, has not been reported before. Most of the intragenic PITX2 mutations are loss-of-function mutations, which result in defective DNA binding or/and decreased transactivation capability of downstream genes [4]. Both PITX2 and FOXC1 are dosage sensitive; the alteration in the level of functional protein (either increased or decreased) is a mechanism of the disease.
The major clinical concern of ARS is glaucoma, which caused serious damage to eyesight, and glaucoma may occur in about $50 \%$ patients with ARS $[4,25]$; the severity of glaucoma correlates with the level of iris inserting into the angle [26]. But only $18 \%$ of the patients with ARS responded to medical or surgical (used solely or in combination) treatment, this may be due to surgical complications such as early fibrosis and the presence of modifier genes [27]. In our study, 
except subject III-3 and subject III-5, the rest all had glaucoma; however, the severities were differential. Subject II1, subject II-2, subject II-3, and subject II-4 were close to blind, and subject III-2 and subject III-4 were well controlled with medicine, while subject III-1 cannot be controlled with medicine.

In summary, we described variable clinical findings in a Chinese family with ARS in this report. They showed variable phenotypes and had no systemic abnormalities. We performed DNA sequence of FOXC1 and PITX2; a homozygous mutation c.1139_1141dupGCG(p.Gly380_Ala381insGly) and a heterozygous mutation c.1359_1361dupCGG(p.Gly456_Gln457insGly) in FOXC1 were identified in all subjects. The mutation (c.-10-30T $>$ C) was identified in PITX2 in subjects III-1 and III-3. Though more functional studies are still needed to prove the association between these mutations and ARS, our results are useful for a better understanding of the spectrum of FOXC1 and PITX2 mutations and provide help for the genetic counseling and gene-specific therapy in the future.

\section{Conflicts of Interest}

The authors declare that there is no conflict of interest regarding the publication of this paper.

\section{Acknowledgments}

The authors thank all the subjects involved in this study.

\section{References}

[1] W. L. Alward, "Axenfeld-Rieger syndrome in the age of molecular genetics," American Journal of Ophthalmology, vol. 130, no. 1, pp. 107-115, 2000.

[2] Y. A. Ito and M. A. Walter, "Genomics and anterior segment dysgenesis: a review," Clinical \& Experimental Ophthalmology, vol. 42, no. 1, pp. 13-24, 2014.

[3] A. Kamińska, A. Sokołowska-Oracz, M. Pawluczyk-Dyjecińska, and J. P. Szaflik, "Variability of clinical manifestations in the family with Axenfeld-Rieger syndrome," Klinika Oczna, vol. 109, no. 7-9, pp. 321-326, 2007.

[4] Z. Tümer and D. Bach-Holm, "Axenfeld-Rieger syndrome and spectrum of PITX2 and FOXC1 mutations," European Journal of Human Genetics, vol. 17, no. 12, pp. 1527-1539, 2009.

[5] B. A. Amendt, E. V. Semina, and W. L. Alward, "Rieger syndrome: a clinical, molecular, and biochemical analysis," Cellular and Molecular Life Sciences, vol. 57, no. 11, pp. 1652-1666, 2000.

[6] R. A. Honkanen, D. Y. Nishimura, R. E. Swiderski et al., "A family with axenfeld-rieger syndrome and Peters anomaly caused by a point mutation (phe112ser) in the foxcl gene," American Journal of Ophthalmology, vol. 135, no. 3, pp. 368375, 2003.

[7] T. A. Hjalt and E. V. Semina, "Current molecular understanding of Axenfeld-Rieger syndrome," Expert Reviews in Molecular Medicine, vol. 7, no. 25, pp. 1-17, 2005.

[8] E. V. Semina, R. Reiter, N. J. Leysens et al., "Cloning and characterization of a novel bicoid-related homeobox transcription factor gene, RIEG, involved in rieger syndrome," Nature Genetics, vol. 14, no. 4, pp. 392-399, 1996.
[9] B. D'Haene, F. Meire, I. Claerhout et al., "Expanding the spectrum of FOXC1 and PITX2 mutations and copy number changes in patients with anterior segment malformations," Investigative Ophthalmology \& Visual Science, vol. 52, no. 1, pp. 324-333, 2011.

[10] M. Tanwar, T. Dada, and R. Dada, "Axenfeld-Rieger syndrome associated with congenital glaucoma and cytochrome P4501B1 gene mutations," Case Reports in Medicine, vol. 2010, pp. 813$822,2010$.

[11] S. Micheal, S. N. Siddiqui, S. N. Zafar et al., "Whole exome sequencing identifies a heterozygous missense variant in the PRDM5 gene in a family with Axenfeld-Rieger syndrome," Neurogenetics, vol. 17, no. 1, pp. 17-23, 2016.

[12] R. Riise, K. Storhaug, and K. Brondum-Nielsen, "Rieger syndrome is associated with PAX6 deletion," Acta Ophthalmologica Scandinavica, vol. 79, no. 2, pp. 201-203, 2001.

[13] R. Riise, B. D'Haene, E. de Baere, K. Grønskov, and K. Brøndum-Nielsen, "Rieger syndrome is not associated with PAX6 deletion: a correction to Acta Ophthalmol Scand 2001: 79: 201-203," Acta Ophthalmologica, vol. 87, no. 8, p. 923, 2009.

[14] D. Kelberman, L. Islam, S. E. Holder et al., "Digenic inheritance of mutations in FOXC1 and PITX2: correlating transcription factor function and Axenfeld-Rieger disease severity," Human Mutation, vol. 32, no. 10, pp. 1144-1152, 2011.

[15] R. A. Saleem, S. Banerjee-Basu, F. B. Berry, A. D. Baxevanis, and M. A. Walter, "Analyses of the effects that diseasecausing missense mutations have on the structure and function of the winged-helix protein foxc1," American Journal of Human Genetics, vol. 68, no. 3, pp. 627-641, 2001.

[16] S. Komatireddy, S. Chakrabarti, A. K. Mandal et al., "Mutation spectrum of foxc1 and clinical genetic heterogeneity of Axenfeld-Rieger anomaly in India," Molecular Vision, vol. 9, pp. 43-48, 2003.

[17] H. J. Yang, Y. K. Lee, C. K. Joo, J. I. Moon, J. W. Mok, and M. H. Park, "A family with Axenfeld-Rieger syndrome: report of the clinical and genetic findings," Korean Journal of Ophthalmology, vol. 29, no. 4, pp. 249-255, 2015.

[18] O. J. Lehmann, J. C. Sowden, P. Carlsson, T. Jordan, and S. S. Bhattacharya, "Fox's in development and disease," Trends in Genetics, vol. 19, no. 6, pp. 339-344, 2003.

[19] P. Challa, "Glaucoma genetics," International Ophthalmology Clinics, vol. 48, no. 4, pp. 73-94, 2008.

[20] F. B. Berry, R. A. Saleem, and M. A. Walter, "FOXC1 transcriptional regulation is mediated by $\mathrm{N}$ - and C-terminal activation domains and contains a phosphorylated transcriptional inhibitory domain," The Journal of Biological Chemistry, vol. 277, no. 12, pp. 10292-10297, 2007.

[21] S. Pierrou, M. Hellqvist, L. Samuelsson, S. Enerbäck, and P. Carlsson, "Cloning and characterization of seven human forkhead proteins: binding site specificity and DNA bending," The EMBO Journal, vol. 13, no. 20, pp. 5002-5012, 1994.

[22] N. Weisschuh, E. De Baere, B. Wissinger, and Z. Tümer, "Clinical utility gene card for: Axenfeld-Rieger syndrome," European Journal of Human Genetics, vol. 19, no. 3, 2011.

[23] J. Kumar and K. Moses, "Transcription factors in eye development: a gorgeous mosaic?” Genes \& Development, vol. 11, no. 16, pp. 2023-2028, 1997.

[24] A. L. Evans and P. J. Gage, "Expression of the homeobox gene Pitx2 in neural crest is required for optic stalk and ocular anterior segment development," Human Molecular Genetics, vol. 14, no. 22, pp. 3347-3359, 2005. 
[25] F. Idrees, D. Vaideanu, S. G. Fraser, J. C. Sowden, and P. T. Khaw, "A review of anterior segment dysgeneses," Survey of Ophthalmology, vol. 51, no. 3, pp. 213-231, 2006.

[26] M. B. Shields, "Axenfeld-Rieger syndrome: a theory of mechanism and distinctions from the iridocorneal endothelial syndrome," Transactions of the American Ophthalmological Society, vol. 81, pp. 736-784, 1983.

[27] M. H. Strungaru, I. Dinu, and M. A. Walter, "Genotypephenotype correlations in axenfeld-rieger malformation and glaucoma patients with foxc1 and pitx2 mutations," Investigative Ophthalmology \& Visual Science, vol. 48, no. 1, pp. 228237, 2007. 


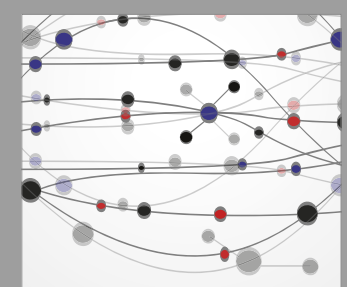

The Scientific World Journal
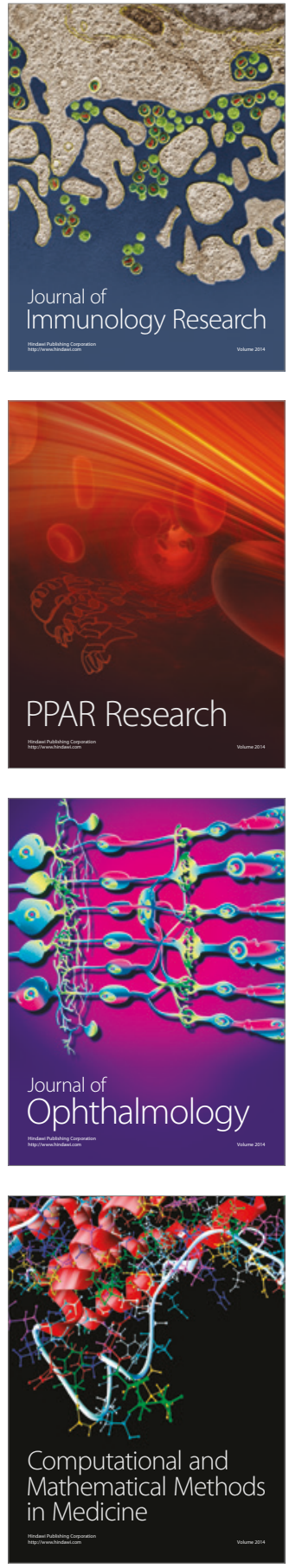

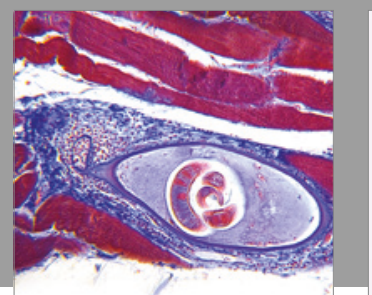

Gastroenterology Research and Practice
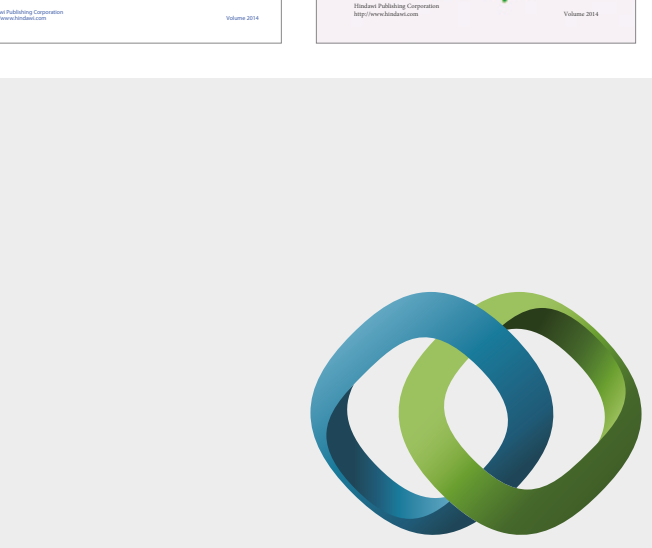

\section{Hindawi}

Submit your manuscripts at

https://www.hindawi.com
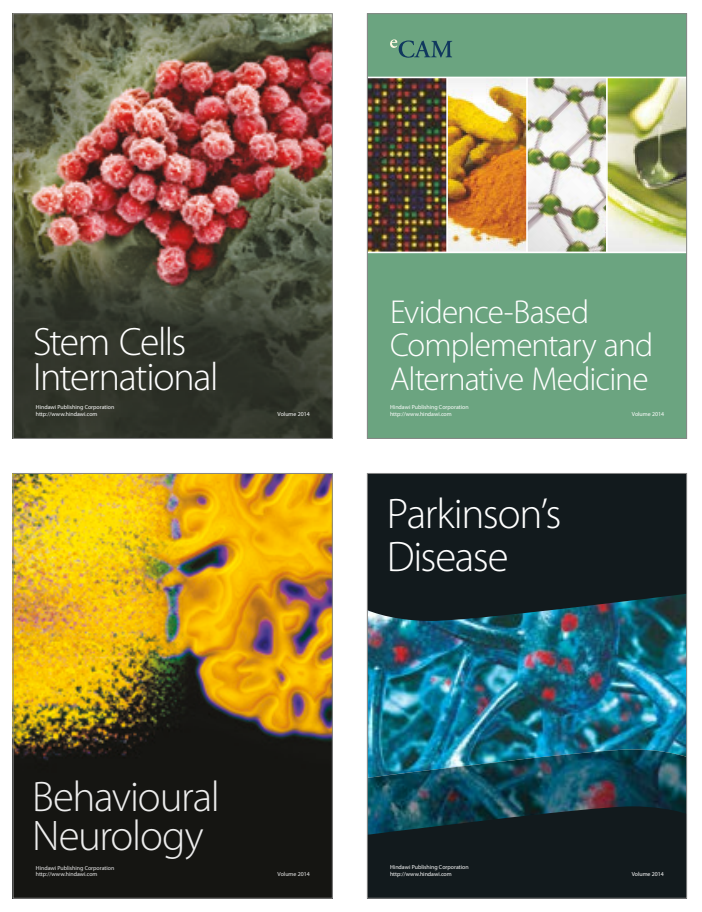
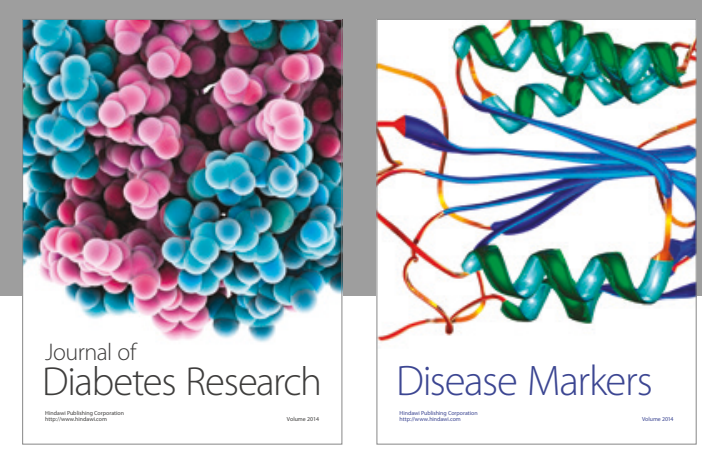

Disease Markers
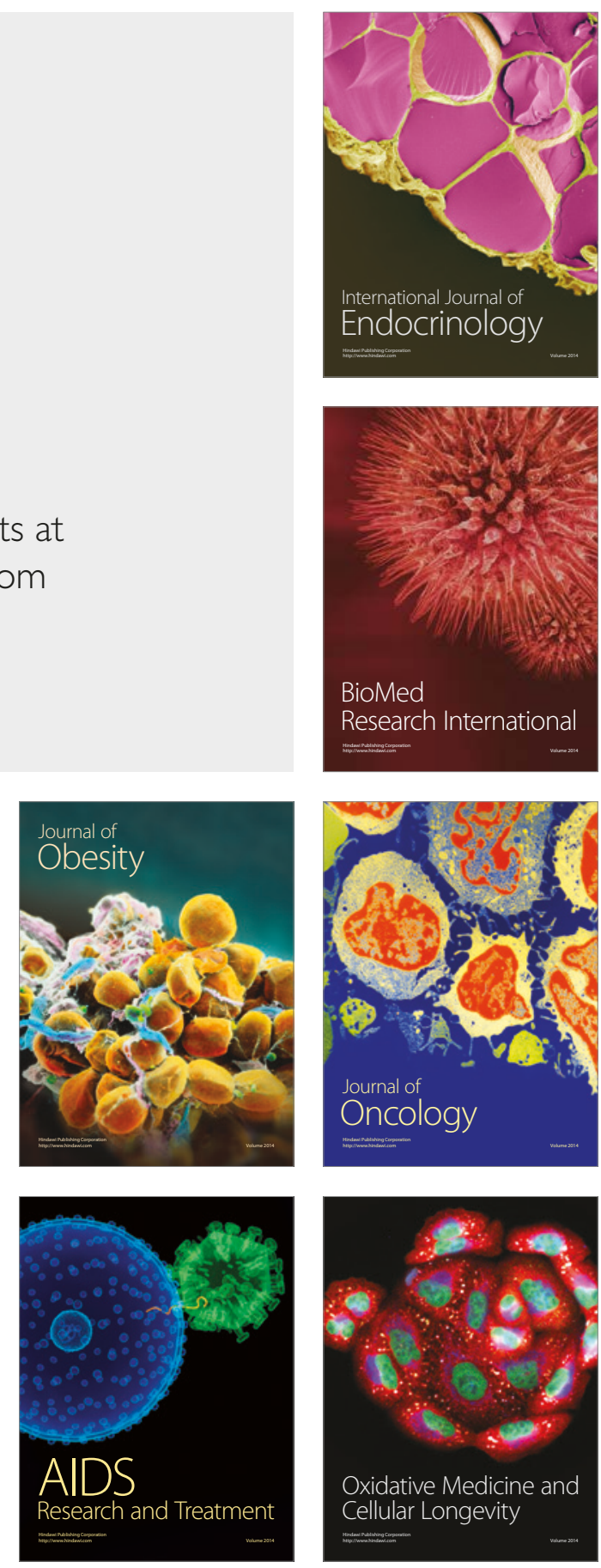